\title{
DETERMINAZIONE DELLA SPINTA DI REAZIONE IN UN CASO SPECIALE.
}

\author{
Nota dell' Ing. CARLO FOSSA MANCINI.
}

§ 1. - Malgrado che le recenti concezioni di meccanica relativistica infirminò la validità rigorosa dei principi della meccanica classica, tuttavia le modificazioni che si dovrebbero introdurre sono talmente piccole che l'accordo fra le deduzioni teoriche ed i risultati sperimentali, per quanto riguarda la meccanica generale e particolarmente la meccanica releste, riesce oltremodo soddisfacente.

Non altrettanto può affermarsi sull'applicazione dei predetti principi all'idrodinamica.

Sappiamo di fatti che in tutti $i$ fenomeni riguardanti questa scienza, si verificano delle azioni secondarie, quali ad esempio le resistenze d'attrito, le quali non possono essere adeguatamente penetrate dall'analisi e debbono perciò essere rappresentate da formule teoretiche nelle quali figurano coefficienti sperimentali. E siccome il valore di questi coefficienti deve essere determinato sperimentalmente, è impossibile con tale.procedimento formare un giudizio esatto sull'accordo fra la teoria e l'esperienza.

Ciò non di meno fra i molti fenomeni idrodinamici che possono formare :ggetto del nostro studio, ve ne ha uno nel quale le predette resistenze non producono alcuna azione sull'effetto finale e perciò si presta bene al raggiungimento del nostro scopo il quale, come ora si disse, consiste nel ricercare l'accordo tra la teoria e l'esperienza.

Questo caso speciale ci viene fornito da un arganetto a reazione, nel quale il liquido in luogo di essere emesso viene aspirato. 
Sul funzionamento di tale apparecchio il prof. Ernesto Mach nella sua rinomata opera $I$ principî della meccanica espost $i$ criticamente e storicamente nel loro stiluppo; così si esprime:

«Si potrebbe supporre che, aspirando del gas, mediante « nua ruota a reazione, la si facesse ruotare in direzione con«traria alla rotazione prodotta dall'uscita del gas. Però in "generale questo non si verifica; come si scorge facilmente « l'aria aspirata dai raggi della ruota deve subito prender parte «al moto dell'apparecchio e si mette così in riposo relativo « rispetto alla ruota; la somma delle aree dell' insieme non può «quindi rimanere nulla. In gewerale l'aspirazione del gas non «produce alcuna rotazione percettibile. Questo fenomeno è ana«logo a quello del non rinculo di un cannone, in cui si in«troduce un proietto. Se si fa comunicare la ruota a reazione «con una palla elastica, munita di un sol tubo di comunica«zione, la quale si comprime periodicamente in modo, che la «stessa quantità di aria sia alternativamente aspirata e poi «emessa si vede che la ruota si muove rapidamente nella «stessa direzione, come se l'aria fosse unicamente emessa. «Questo fenomeno è dovuto in parte al fatto che l'aria aspi« rata che partecipa subito al moto della ruota, non può pro« durre alcuna rotazione di reazione, ed in parte alla diffe« renza del moto dell'aria esterna, secondo che l'aria esce o «entra per le ruote. Quando l'aria è emessa, essa esce per i « raggi con un moto di rotazione; quando ̀̀ aspirata, essa si «precipita da tutte le parti senza rotazione verso l'apertura ».

Per quanta deferenza si voglia prestare all'autorita dell'illustre professore è ben difficile che il lettore si accontenti di una spiegazione così incounpleta e così poco rigorosa, è impossibile che per mezzo di questi soli ragionamenti egli si persuada pienamente che il fluido che attraversa l'apparecchio non esercita sul medesimo aleuna azione e perciò questo devo rimanere immobile come è confermato dall'esperienza. E necessario perciò penetrare più a fondo nell'argomento e dare del fatto stesso una dimostrazione più soddisfacente. 
§ 2. - Nello studio di una macchina a reazione possiamo procedere in due modi differenti. Possiamo considerare la macchina in azione e misurare il lavoro da essa prodotto, ovvero possiamo considerare la macchina ferma e misurare la spinta di reazione. In questo secondo caso la macchina predetta si trasforma in una bilancia a reazione.

Tenuto conto che questo secondo procedimento è assai più semplice e capace di maggior precisione, noi ci atterremo al esso. Benchè quanto siamo per dire possa convenire ad un fluido qualsiasi, ciò non dimeno per amore di semplicità ed in vista delle esperienze che ci proponiamo di fare, considereremo soltanto un liquido a densità costante quale l'acqua.

Nel caso in cui il liquido esce dall'apparecchio, la bilancia a reazione prende una forma semplicissima quale vedesi rappresentata schematicamente nella fig. 1 .

A è il fulcro della bilancia, B l'orificio di efflusso da cui l'acqua esce sotto forma di un getto orizzontale filiforme parallelo all'asse di proiezione $\mathrm{XX}$. $\mathrm{P}$ è il peso che per mezzo di un filo e di una carrucola fa equilibrio alla spinta di reazione.

$\mathrm{Si}$ ritiene che il pelo libero $\mathrm{C}$ nell'interno del recipiente, per mezzo di adeguata alimentazione venga mantenuto costantemente allo stesso livello di modo che l'effusso abbia luogo con regime permanente.

l'er determinare la spinta di reazione facciamo uso del teorema delle quantita di moto proiettate su di un asse espresso clalla formola

$$
\frac{d \mathbf{Q}}{d t}=\int \mathrm{F}_{e} k d \tau+\int \mathrm{P}_{e} d \sigma
$$

nella quale $Q$ ̀̀ il risultante delle quantità di moto proiettate sull'asse, $F_{e}$ la forza esterna riferita all' unità di massa, $P_{e}$ la pressione riferita all' unità di superficie amberlue proiettate sull'asse, $k$ la densità dell'acqua, $d \tau$ e $d$ o gli elementi di volume e di superficie, $t$ il tempo.

Consideriamo la massa idrica contenuta nell'apparecchio, nelle posizioni successive $\mathrm{CBBC}, \mathrm{C}^{\prime} \mathrm{B}^{\prime} \mathrm{B}^{\prime} \mathrm{C}^{\prime}$ che essa assume 
ai tempi $t$ e $t+t$. Ora a causa del moto permanente, nel quale le velocità sono indipendenti dal tempo, la porzione dí massa contenuta nello spazio $\mathrm{C}^{\prime} \mathrm{BBC}^{\prime}$ durante il tempuscolo $d t$ non subisce alcuna variazione di velocità. Tale variazione compete soltanto alle masse che occupano gli spazi $\mathrm{CO}^{\prime} \mathrm{C}^{\prime} \mathrm{C}$, B B' $\mathrm{B}^{\prime} \mathrm{B}$. Diciamo $w$ e $c$ la velocità e l'area della sezione in $e^{*}$
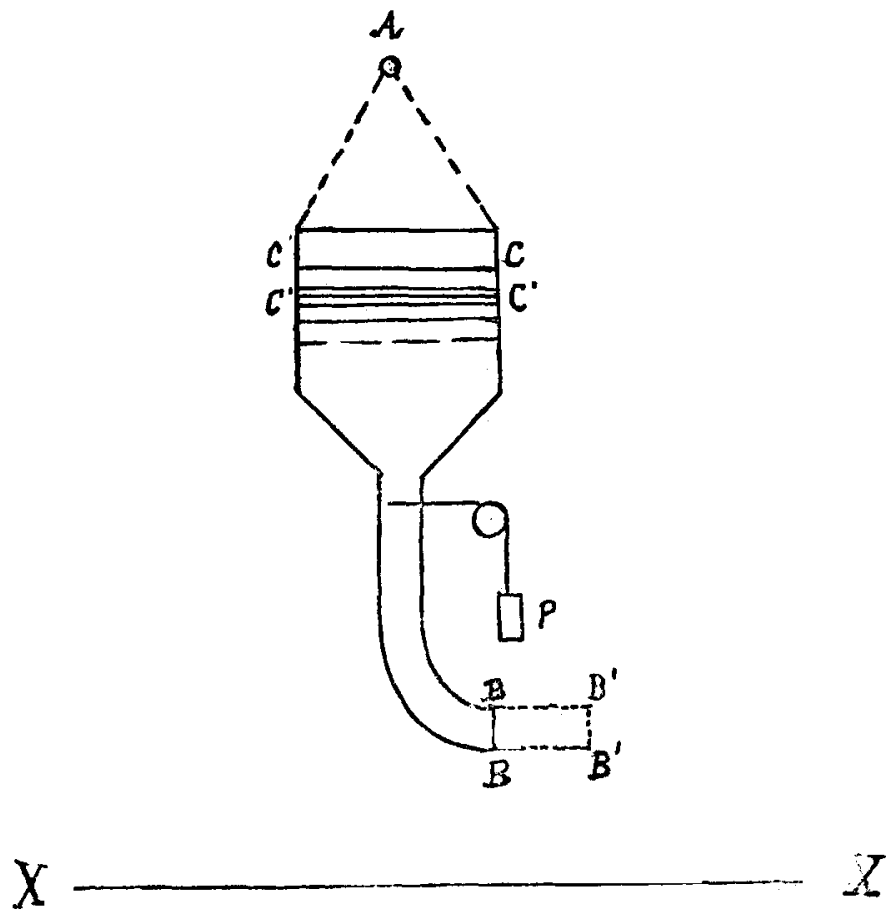

Fig. 1.

$v$ e $b$ la velocitàe l'area della sezione in B. Dall'ispeziono della figura e per quanto si disse in antecedenza risulta

$$
\mathrm{CO}^{\prime}=v d t \quad ; \quad \mathrm{BI} \mathrm{B}^{\prime}=v d t .
$$

Donde segue che le masse, le quali occupano gli spazi CC'C'C, B B B B saranno espresse da $k c w d t, k b v d t$ e le rispettive quantità di moto da

$$
k c v^{2} d t, \quad k b v^{2} d t
$$


Proiettando tali grandezze sull'asse XX e tenendo conto che la $v$ è parallela e la $w$ è normale all'asse, si ottiene

$$
d \mathbf{Q}=k \cdot b v^{2} d t
$$

1alla quale dednciamo immediatamente il valore del primo membro dell'equazione (1)

$$
\frac{d \mathrm{Q}}{d t}=k b v^{\gamma}=\frac{\tilde{\omega}}{g} b v^{2}
$$

essendo $\tilde{\omega}$ il peso specifico dell'acqua e $g$ l'accelerazione dovuta alla gravità.

Quanto al primo termine $\int \mathrm{F}_{e} k \partial \tau$, che figura nel secondo membro dell'equazione (1.); osserviamo che, essendo la gravità l'unica forza esterna che sollecita la massa idrica si ha $\mathbf{F}_{e}=g \cos \alpha$, dove $\alpha$ è l'angolo di inclinazione della forza sul l'asse di proiezione, e siccome quest'angolo è retto per tutti gli elementi, $F_{e}$ è costantemente nulla e per conseguenza il valore di $\int \mathbf{F}_{e} k d \tau$ ̀̀ nullo. Il secondo termine della (1) ̀̀ rappresentato da un integrale di superficie $\int \mathrm{P}_{e} d \sigma$ che va esteso a tutto il contorno chiuso che comprende la massa considerata.

Se diciamo $P_{a}$ la pressione atmosferica, che si ritiene uniforme, si ha evidentemente

$$
\int P_{a} d \sigma=0 \text {. }
$$

Possiamo perciò in luogo di $f \mathrm{P}_{a} d \sigma$ scrivere

$$
\int \mathbf{P}_{e} d \sigma-\int \mathbf{P}_{a} d \sigma \text {. }
$$

Indichiamo con $\sigma^{\prime}$ la superficie dell'apparecchio bagnata Aall'acqua, con $\sigma^{\prime \prime}$ la superficie libera, sarà

$$
\sigma=\sigma^{\prime}+\sigma^{\prime \prime}
$$

e per conseguenza

$$
\int \mathbf{P}_{e} d \sigma-\int \mathbf{P}_{a} d \sigma=\int\left(\mathbf{P}_{e}-\mathbf{P}_{a}\right) d \sigma^{\prime}+\int\left(\mathbf{P}_{e}-\mathbf{P}_{a}\right) d \sigma^{*} .
$$

Is siccome sulla superficie libera $\mathrm{P}_{e}=\mathbf{P}_{a} ; \int\left(\mathbf{P}_{e}-\mathbf{P}_{a}\right), d \sigma^{\prime \prime}=0$ donde

$$
\int \mathrm{P}_{e} d \sigma=\int\left(\mathrm{P}_{e}-\mathrm{P}_{a}\right) d \sigma^{\prime}
$$

Ora quest'ultimo integrale esteso alla superficie dell'apparecchio bagnata dall'acqua ci rappresenta la spinta di reazione secondo 
l'asse di proiezione, ossia la grandezza che nella bilancia a reazione è equilibrata dal peso. Indicandola con S e scegliendo quale direzione positiva quella contraria alla velocità di efflusso si ottiene dalla (1)

$$
\mathrm{S}=\int \mathrm{P}_{e} d \sigma:=\frac{\tilde{\omega}}{\mathrm{g}} b v^{2}
$$

§ 3. - Ed ora passiamo al caso in cui il liquido, in luogo di essere emesso venga aspirato.

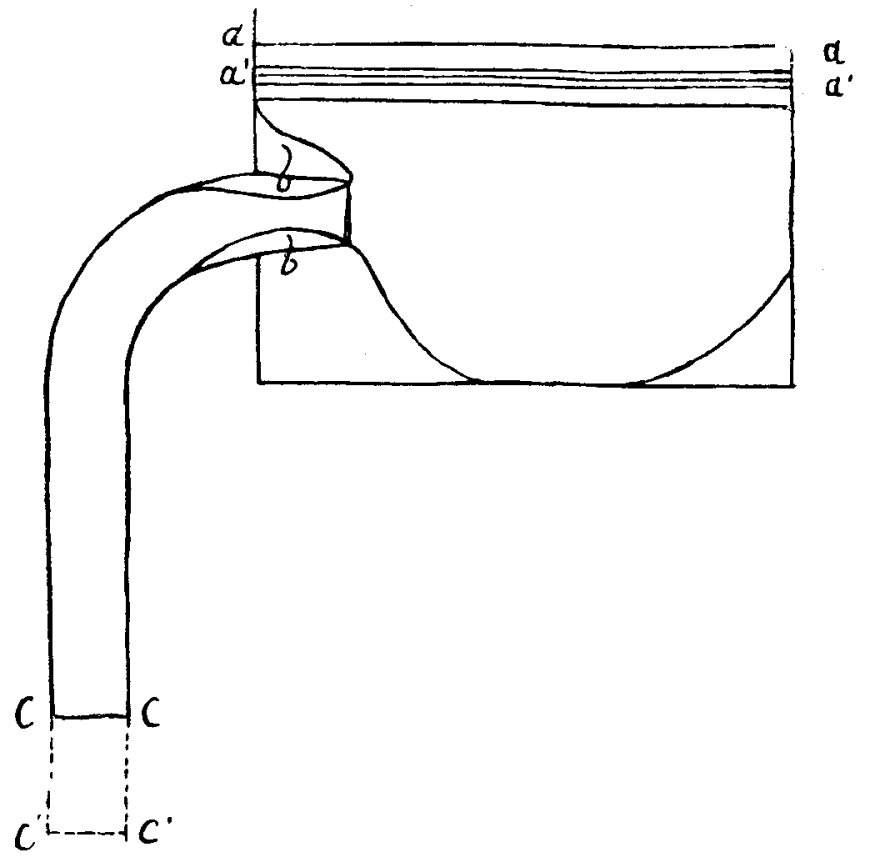

Fig. 2 .

In questo caso l'apparecchio prende la forma indicata schematicamente nella fig. 2. A causa della depressione che si verifica all'orificio d'imbocco, i filetti assumono una disposizione speciale quale vedesi nella predetta figura.

La massa idrica in moto, alla quale dobbiamo applicare il principio delle quantità di moto proiettate, occupa al tempo $t$ lo spazio abccba, al tempo $t+d t$ lo spazio $a^{\prime} b c^{\prime} c^{\prime} b a^{\prime}$. 
In conseguenza del regime permanente, cui per ipotesi soddisfa il moto del liquido, la variazione della quantità di moto subita dall' intera massa nel tempo infinitesimo $d t$ è data, per quanto si disse in antecedenza, dalle variazioni che nello stesso tempo subiscono le masse $a a^{\prime} a^{\prime} a, c c^{\prime} c^{\prime} c$; e siccome queste masse sono animate da velocità verticali, la somma delle variazioni della quantità dı moto proiettata sull'asse orizzontale risulta nulla e per conseguenza parimenti nulla è la spinta orizzontale di reazione che il liquido esercita sull'apparec. chio.

Questo fatto è generale e si verifica sempre qualunque sia la forma dell'orificio di imbocco. Evidentemente col variare della forma dell'orificio di imbocco varia anche la disposizione dei filetti idrici ma, purchè siano rispettate le condizioni di efflusso sovra esposte, la spinta orizzontale di reazione è costantemente nulla.

§ 4. - Supponiamo ora che l'orificio d' imboceo sia formato da un tubo cilinilrico a sezione circolare costante su tutta la sua lunghezza.

La chiamata che si produce tutto attorno all'imboceo fa si che i filetti, i quali convergono verso l'asse del tubo, prendano presso a poco la forma rappresentata dalla fig. 3. Dalla considerazione di questa figura dednciamo immediatamente che la vena idrica nell' interno del tubo debba presentare una sezione contratta.

Alla sezione contratta, la velocità è massima. Allontanandosi da questa sezione e procedendo in senso contrario al moto del liquido, la velocità va mano mano diminnendo di modo che se le dimensioni del recipiente, come si suppone sono assai grandi di fronte alla sezione del tubo; la velocità dell'acqua che lambisce le pareti del recipiente è del tutto trascurabile e la pressione si distribuisce secondo la legge idrostatica. Donde segue che il recipiente, preso a sè, non è soggetto ad alcuna spinta di reazione e questa si esercita soltanto sul tubo di efflusso. 
Diciamo dunque $b$ l'area della sezione contratta, $a$ l'area della sezione normale del tubo ed applichiamo alla massa idrica baccab il solito teorema delle quantità di moto proiettato.

In questo caso il termine $\int P_{e} d \sigma$, che va esteso al contorno chiuso della massa che si considera, si divide in tre parti ; la

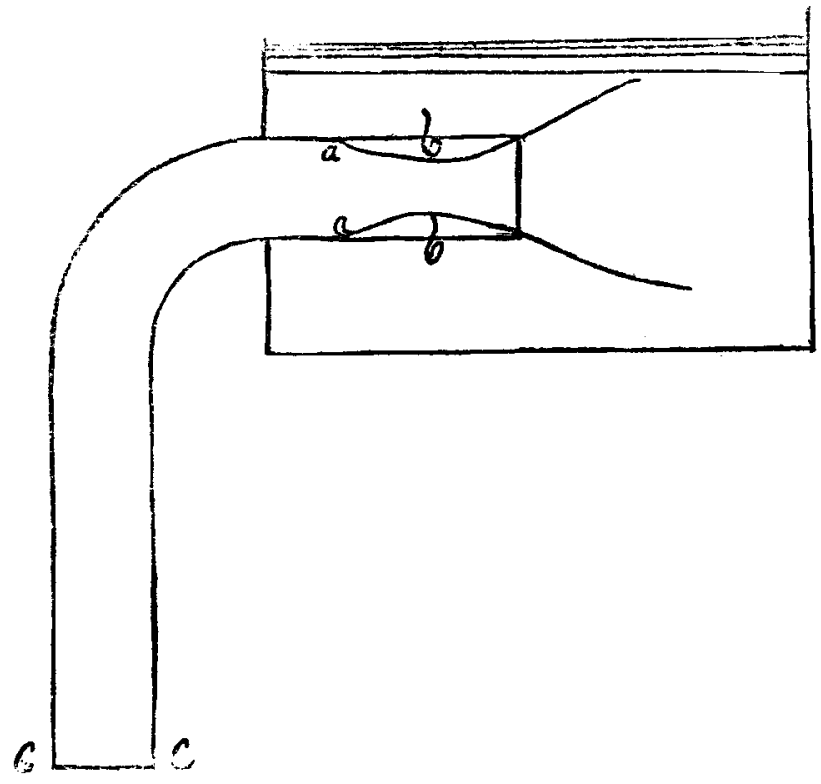

Fig. 3.

prima riguarda la superficie o dell'apparecchio bagnato dal liquido, la seconda spetta alla superficie di sbocco $c c=\sigma^{\prime \prime}$, la terza si riferisce all'imboceo $a b b a=\sigma^{\prime \prime \prime}$, si ha perciò

$$
\int \mathbf{P}_{e} d \sigma=\int \mathbf{P}_{e} d \sigma^{\prime}+\int \mathbf{P}_{e} d \sigma^{\prime \prime}+\int \mathbf{P}_{e} d \sigma^{\prime \prime} .
$$

Sia $P_{a}$ la pressione atmosferica, per quanto si disse in antecedenza $\int \mathrm{P}_{a} d \sigma=0$ e quindi

$$
\begin{gathered}
\int \mathbf{P}_{e} d \sigma=\int \mathbf{P}_{e} d \sigma-\int \mathbf{P}_{a} d \sigma \\
\int \mathbf{P}_{e} d \sigma=\int\left(\mathbf{P}_{e}-\mathbf{P}_{a}\right) d \sigma^{\prime}+\int\left(\mathbf{P}_{e}-\mathbf{P}_{a}\right) d \sigma^{\prime \prime}+\int\left(\mathbf{P}_{e}-\mathbf{P}_{a}\right) d \sigma^{\prime \prime} \text { che, }
\end{gathered}
$$


tenuto conto della direzione della pressione dovremo scrivere

$$
\int \mathbf{P}_{e} d \sigma=\int\left(\mathbf{P}_{e}-\mathbf{P}_{a}\right) d \sigma^{\prime}+\left(\int \mathbf{P}_{e}-\mathbf{P}_{a}\right) d \sigma^{\prime \prime}-\int\left(\mathbf{P}_{e}-\mathbf{P}_{a}\right) d \sigma^{\prime \prime \prime} .
$$

Ora il primo termine del secondo membro rappresenta la spinta di reazione $S$ esercitata dal liquido sull'apparechio, il secondo è nullo per essere śu tutta la superficie $\sigma^{\prime \prime}, P_{e}=P_{a}$. Quanto al significato del terzo termine

$$
\int\left(\mathrm{P}_{e}-\mathrm{P}_{a}\right) d \sigma^{\prime \prime \prime}
$$

osserviamo che $P_{a}-P_{e}$ rappresenta la depressione esistente nella sezione contratta $b b$, la quale depressione è costante su tutta la superficie $a b b a$. Indicando con $h$ il valore in colonna d'acqua di tale depressione, ed essendo a l'area della seziono normale del tubo. si ha

$$
\int\left(\mathbf{P}_{a}-\mathbf{P}_{e}\right) d \sigma^{\prime \prime \prime}=\tilde{\omega} a h
$$

Sostituendo nella (1)

londe

$$
\frac{\tilde{\omega} b}{g} v^{2}=\int \mathrm{P}_{e} d \sigma=\mathrm{S}+\tilde{\omega} a h
$$

$$
\mathrm{S}=\frac{\tilde{\omega} b v^{2}}{g}-\tilde{\omega} a h
$$

Analizzando questa formola troviamo che essa si compone di due ternini, il primo rappresenta l'azione cinetica dell'acqua nell'interno dell'apparechio, il secondo la depressione all'imbocco. E siccome, per quanto si è detto in antecedenza la spinta totale è sempre nulla, si giunge alla conclusione che il liquido aspirato produce sull'apparecehio due azioni eguali e contrarie che si elidono reciprocamente.

Queste conclusioni dedotte dallo studio della bilancia di reazione valgono egualmente per l'arganetto, ossia pel caso trattato dal prof. Mach, e ci forniscono la spiegazione rigorosa e completa del motivo per il quale il fluido non esercita alcuna azione sull'apparecchio e perciò questo rimane immobile come è confermato dall'esperienza. 
§ 5. - Ma la formola (3) si presta anche ad un altro scopo. Essa ci permette di trovare il coefficiente di contrazione della vens, ossia di determinare il rapporto $\frac{b}{a}$ tra l'area della sezione contratta e quella della sezione del tubo.

Consideriamo la sezione contratta come un orificio alimentato da un recipiente molto grande nel quale il liquido si mantiene a livello costante.

Trascurando le resistenze d'attrito e la velocità di arrivo dell'aeqna di alimentazione, pel teorema di Torricelli abbiamo

$$
h=\frac{v^{2}}{2 g} .
$$

Sostituendo nella (3) otteniamo

$$
\mathrm{S}=\frac{\tilde{\omega}}{g} v^{2}\left(b-\frac{a}{2}\right)
$$

Ora per quanto si disse in antecedenza, la spinta di reazione deve essere costantemente nulla, perciò dovrà essere

$$
\frac{b}{a}=\frac{1}{2}
$$

Ossia, in altri termini, il coefficiente di contrazione della vena in questo caso speciale è eguale ad un mezzo.

Questo risultato non costituisce una novità e poteva essere preveduto. Ed in vero osservando il moto dell'acqua prodotto dall'aspirazione del sifone, si trova che esso è completamente analogo a quello che si verifica in un recipiente sulla parete interna del quale sia stato fissato il tubo addizionale di Borda. E siccome è stato dimostrato tanto teoricamente che sperimentalmente che per questo tubo il coefficiente di contrazione è eguale ad un mezzo, si giunge alla conclusione che anche. nel caso da noi trattato detto coefficiente debba avere lo stesso valore. 
$\S 6$. - Ed ora veniamo alla parte sperimentale. Nel caso in cui il liquido esce da un orificio $b$ con velocità $v$ la spinta li reazione per la formola (2) del $\S 2$ è

$$
\mathrm{S}=\frac{\tilde{\omega}}{b} b v^{2}
$$

Questa formola non contiene alcun coefficiente sperimentale e sembrerebbe per ciò adattata al confronto tra l'esperienza e la teoria. Devesi però ricordare che essa si basa su di una ipotesi approssimativa, sull' ipotesi cioè clıe all'orificio di sbocco i filetti idrici abbiano tutti la stessa velocità $v$. In realtà, a cansa dell'attrito, i filetti centrali hanno una velocita maggiore dei filetti periferici per cui la formola esatta dovrebbe scriversi

$$
\mathrm{S}=\frac{\tilde{\omega}}{g} \int v^{2} d b={ }_{g}^{\tilde{\omega}} \lambda b v_{e}{ }^{2}
$$

dove $v_{e}$ è la velocità effettiva e $\lambda$ un coefficiente sperimentale, maggiore dell' unità, finzione delle resistenze d'attrito.

Si vede quindi che questa formola non è adatta allo scopo. Ciò non di meno tenuto conto che gli effetti prodotti dalle resistenze d'attrito sono in questo caso molto piccoli, si può in via d'approssimazione ritenere $\lambda=1$ e confrontare $\mathrm{i}$ valori della spinta dati dalla (2) con quelli ottenuti in via sperimentale.

Da alcune esperienze da me eseguite nel $1898^{\prime}$ ') su di nna bilancia a reazione nella quale l'orificio di efflusso aveva un diametro di quattro millimetri si ottennero i seguenti risultati.

') Ing. Carlo Fossa-Mancini. - Contributo allo studio dei getti trasciuanti. - L' Ingegneria eivile e le arti industriali, vol. XXIII ; 'Torino, Camilla e Bertolero. - (In questa Memoria fu scritto per errore che il diametro dell'orificio era di tre millimetri). 
Portata in litri al secondo dell'orificio di efflusso 0,05 . spinta di reazione in grammi

$$
\frac{79.5}{4}=19,875 \text {. }
$$

Scegliamo come unità di misura per le lunghezze il centinetro, per le forze il grammo. Cio posto il peso specifico dell'acqua, ossia il peso del centimetro cubo risulta $\tilde{\omega}=1$, il diametro dell'orificio di eftlusso $\partial=\mathbf{0 , 4}$, la portata del medesimo in grammi $q=50$ e l'accelerazione dovuta alla gravità $g=981$.

Essendo $b$ la sezione dell'orificio di efflusso si ha

$$
b=\frac{\pi d^{2}}{4}=0,1257 ; \quad v=\frac{q}{b}=\frac{50}{0,1257}=397.8 .
$$

Sostituendo questi dati nella formola

$$
\mathrm{S}=\frac{\tilde{\omega}}{g} b v^{2}
$$

si ottiene $\mathrm{S}=20,28$ grammi, risultato che si accorda abbastanza bene con quello sperimentale sovra riportato.

La differenza $20,28-19,86=0,42 g r$, che per essere $\lambda>1$ dovrebbe venire leggermente aumentata, devesi attribuire agli errori di osservazione relativi alla misura della spinta, della portata e del diametro dell'orificio di efflusso, i quali errori, in considerazione del modo rudimentale con' il quale venivano eseguite l'esperienze, non potevano certamente essere trascurati.

§ 7. - Ma l'apparecchio che meglio si presta allo scopo è la bilanıia a reazione nella quale il liquido in luogo di essere emesso è aspirato.

In questo caso, come già è stato dimostrato, la spinta di reazione è costantemente nulla e, pel confronto tra l'esperienza e la teoria non occorre fare alcuna misura, basta constatare che la bilancia di reazione rimane immobile, ossia non indica alcuna spinta, quando l'apparecchio è attraversato dall'acqua. 
Alla bilancia a reazione possono essere assegnate svariate forme. Nell' intento di usufruire alcune parti dell'apparecchio di cui mi ero servito per altre esperienze ho dato ad essa la

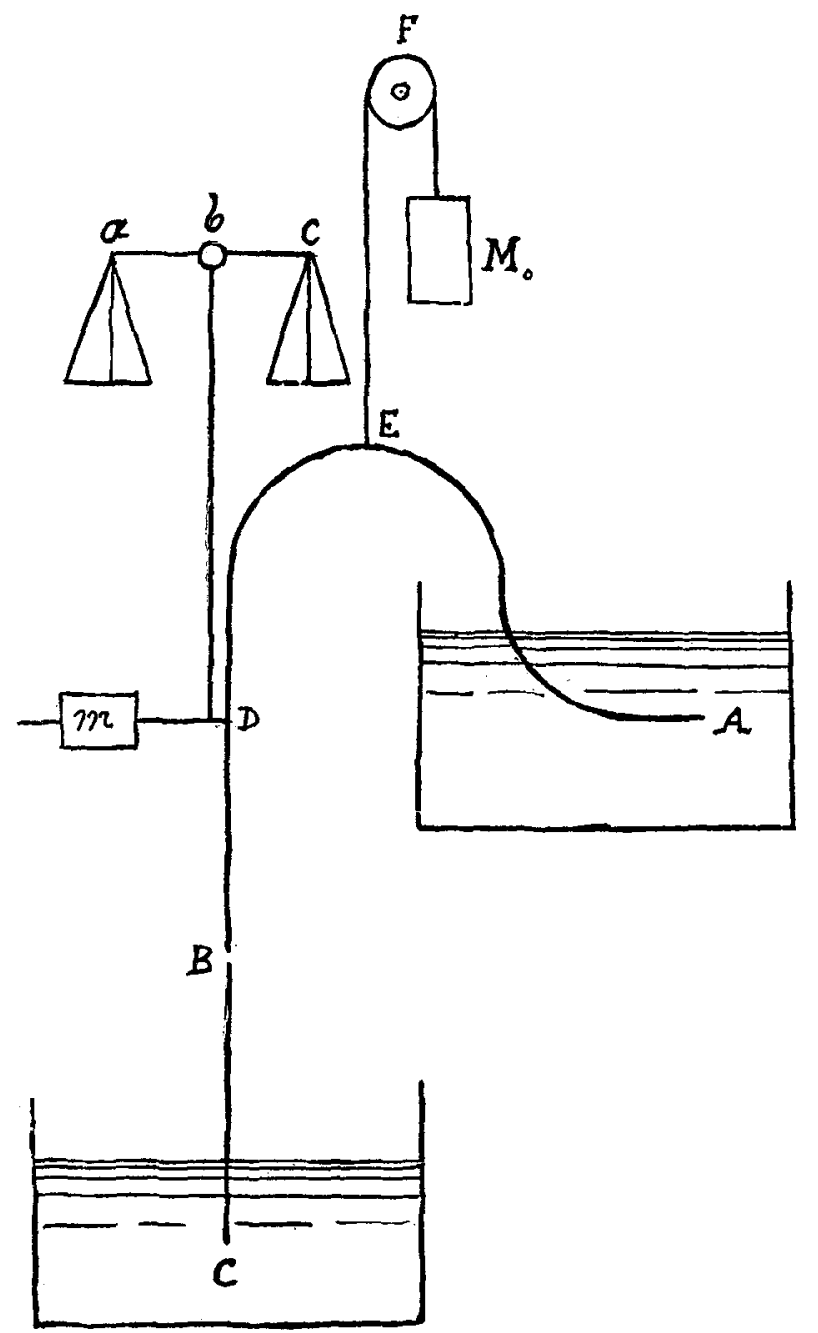

Fig. 4.

forma rappresentata schematicamente dalla fig. 4. Questa forma richiede un preventivo adescamento che per mezzo di una disposizione piu conveniente potrebbe essere evitato. 
Un tubo-sifone ABC lascia fluire l'acqua dal serbatoio $\mathbf{A}$ al serbatoio $O$, i cui peli per mezzo di opportnne disposizioni vengano mantenuti a livello costante. La parte AB del sifone è mobile e può subire un piccolo spostamento relativamente alla parte fissa $\mathrm{BC}$ senza incontrare resistenze apprezzabili. L' uuione ermetica fra le due parti viene ottenuta per mezzo di un giunto flessibile, che permette tale spostamento.

Nella posizione di equilibrio il ramo discendente del sifone D B ̀̀ verticale e l'asse geometrico dell'orificio d'imbocco orizzontale.

Sotto l'azione della spinta di reazione la parte mobile del sifone ruota di un angolo piccolissimo attorno al punto $\mathrm{B}$ ed in conseguenza di questa rotazione il punto I) descrive un piccolissimo arco di cerchio. Questo movimento per mezzo del braccio $\mathrm{J} b$ si trasmette al giogo $a c$, girevole attorno al fulcro $b$ * portante i piattini della bilancia. Sulluno e sull'altro di questi piattini, a seconda del senso nel quale si produce la rotazione si colloca il peso destinato ad equilibrare e misurare la spinta di reazione.

A fine di dare alla bilancia la necessaria stabilità, la parte mobile del sifone è sostenuta superiormente da una funicella EF $\mathrm{M}_{0}$. Questa funicella parte dal punto $\mathrm{E}$ passa sulla gola della carrucola $F$ e termina al contrappeso $M_{0}$. Il peso $M_{0} \grave{e}$ alquanto superiore al peso $M_{1}$ della parte mobile BEA. La grandezza $M_{0}-M_{1}$ rappresenta il grado di sensibilità della bilancia. Questi gradi possono variace ad libitum entro limiti assai estesi.

Nella posizione di equilibrio vale a dire quando la spinta di reazione ̀̀ nulla, il filo EF, è verticale ed il contrappeso $M_{0}$ trovasi al punto pià basso della sua corsa. Quando la spinta di reazione assume un valore finito, il filo EF si allontana dalla verticale ed il contrapoeso si innalza. Il fatto che il contrappeso si innalza in liogo di abbassarsi dimostra che l'equilibrio della bilancia è stabile. 
§ 8. - Per eseguire con questa bilancia l'esperienza di controllo di cui ci stiamo occupando, si comincia coll'adescare il sifone. Quindi spostando il contrappeso scorrevole $m$ si porta a zero l'indice della bilancia. Ciò fatto si apre il rubinetto che chiude l'orificio di efflusso $\mathrm{C}$.

Subito si produce una depressione nell'interno del sifone, la quale si propaga rapidamente all'orificio di imbocco.

Per effetto di questa depressione la bilancia si sposta bruscamente da sinistra a destra, dando luogo ad una serie di oscillazioni gradatamente decrescenti. Intanto il liquido contenuto nel sifone sotto l'azione della gravita si mette in moto ed in breve raggiunge la velocità di regime. A questo istante l'azione cinetica dell'acqua eguaglia la depressione e la bilancia, non essendo più sollecitata da alcuna forza, ritorna allo zero.

§ 9. - Per determinare il grado di precisione del metodo predetto confrontiamo la spinta massima che l'apparecchio proflurebbe so sn di esso si esercitasse soltanto l'ázione cinetica dell'acqua, con la minima spinta che la bilancia è al caso di registrare.

L'azione cinetica dell'acqua, per quanto è stato detto nel $\S 4$ è data da

$$
\frac{\tilde{\omega}}{g} b v^{2}
$$

nella quale $b$ è l'area della sezione contratta $\theta v$ la velocita con la quale il liquido l'attraversa.

Per la determinazione di $b$ osserviamo che essendo il coefficiente di contrazione eguale ad $\frac{1}{2}$ si ha $b=\frac{a}{2}$ dove $a$ ì l'ares dell'orificio d'imbocco. Quanto alla velocità $v$, la si ottiene facilmente dalla portata $q$ del sifone per mezzo della formola

$$
v=\frac{q}{b}
$$

Nel nostro caso scegliendo, come già si fece in antecedenza, per unità di lunghezza il centimetro e per unità di forza il 
grammo, ed essendo il diametro del tubo $d=1,3$ e la portata $q=333$ grammi si la

$$
\begin{gathered}
\tilde{\omega}=1 ; g=981 ; a=\frac{\pi d^{2}}{4}=1.372 \\
b={ }_{2}^{a}=0,686 \quad: \quad v=\frac{333}{0,686}=485.4 \\
\frac{\tilde{\omega}}{g} b v^{2}=164.8 \text { grammi. }
\end{gathered}
$$

La minima spinta che nella bilancia a reazione da me adoperata, prodnce uno spostamento apprezzabile dell'indice, è di circa un grammo. Così che anche con una bilancia costruita in modo primitivo ed ultra grossolano, quale è quella di cui mi sono servito, l'errore massimo di cui possono essere affette le misure non è supesiore ad $\frac{1}{150}$.

Ma io non ho voluto far altro che mostrare la via che conduce allo scopo e wi anguro che qualche fisico volonteroso, il quale si trova in possesso di un buon Gabinetto, voglia ripetere l'esperienza, facendo tesoro dei mezzi perfezionati di cui dispone la tecnica di oggi giorno. Il metodo proposto, che sotto un certo punto di vista ha molta analogia con quello che gli Elettrotecnici chiamano metodo di riduzione allo zero, si presta senza dubbio ad un alto grado di precisione.

Castelplanio, 24 maggio 1918. 